\title{
Yttrium-90 Radioembolization of Metastatic Adrenocortical Carcinoma to the Liver following Systemic Chemotherapy and Surgical Resection of the Primary Lesion
}

\author{
Nicholas Pigg Daniel Aboubechara Roberto Fourzali Brian Baigorri \\ Department of Radiology, Aventura Hospital and Medical Center, Aventura, FL, USA
}

Keywords

Yttrium-90 radioembolization - Adrenocortical carcinoma $\cdot$ Hepatic metastases

\begin{abstract}
Use of yttrium-90 (Y-90) is used for primary liver tumors and a handful of liver metastatic lesions. Y-90 treatment for metastatic adrenocortical carcinoma (ACC) to the liver is currently off-label, with one previously documented case report. In this case report, we present a 52-year-old woman with ACC and extensive liver metastatic disease. After failed chemotherapy, multidisciplinary discussion suggested potential Y-90 treatment for palliative purposes. After undergoing Y-90 treatment separately to the right and then the left hepatic lobes, subsequent patient visits demonstrated significantly improved clinical function as well as complete radiographic resolution of liver metastatic disease mainly from ACC. This case report demonstrates the potential efficacy of $\mathrm{Y}-90$ for off-label uses in liver metastatic disease. This case and similar cases may open the door to a wide variety of potential indications for $Y-90$ treatment.

\section{Introduction}

The use of yttrium-90 (Y-90) to treat primary and metastatic lesions within the liver has been researched since 1960 due to the lack of effective treatment options. In the 1990s the use of glass and resin microspheres was developed to deliver treatment directly to the lesion [1]. Historically Y-90 has been approved for the treatment of hepatocellular carcinoma and 


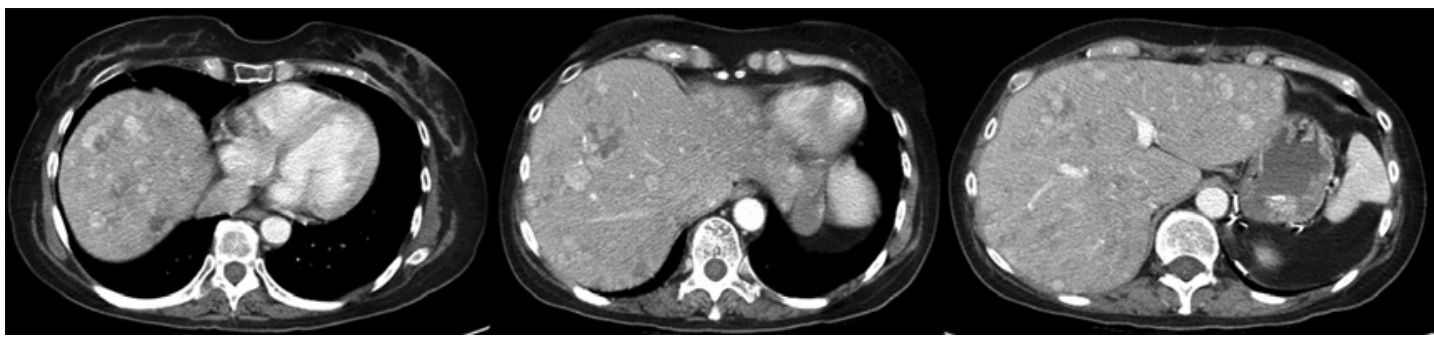

Fig. 1. Axial contrast-enhanced CT images revealing more than 50 hypodense lesions throughout the liver. The largest of these measured approximately $3.4 \times 3.5 \mathrm{~cm}$. The adrenal mass has been surgically removed.

metastatic colorectal cancer to the liver. However, recently there has been investigation into alternative off-label uses for Y-90, such as radiation segmentectomy and radiation lobectomy, and for the treatment of unconventional hepatic metastases such as breast cancer, melanoma, pancreatic cancer, lung cancer, cholangiocarcinoma, and renal cell carcinoma [2]. One type of lesion that has not been well studied is adrenocortical carcinoma (ACC) with metastasis to the liver.

ACC is a rare malignancy with a poor prognosis and a poorly understood pathogenesis. At diagnosis it is often large in size, has metastasized, and is accompanied by multiple paraneoplastic conditions [3]. These syndromes usually involve excess steroid molecules and often present with the clinical features of Cushing's syndrome. The currently available treatment options for ACC are limited and include surgical removal, mitotane with or without streptozotocin, thermal radiofrequency ablation, and tumor bed radiation [3]. The treatment of metastatic lesions is far more limited and has low success rates. The use of Y-90 to treat adrenal metastases to the liver is off-label and has only been documented once. Within that study, metastatic ACC was treated by combining locoregional Y-90 therapy for bilobar liver disease with first-line etoposide, doxorubicin, and cisplatin plus mitotane (EDP-M) chemotherapy and debulking surgery [4]. The treatment allowed the patient to remain recurrence free for 12 months.

In this case study, we present a patient with ACC and chemotherapy-resistant metastatic disease to the liver with a complete clinical response to bilobar Y-90 treatment, as well as a complete radiologic response.

\section{Case}

The patient is a 52-year-old woman with Cushing's syndrome, uncontrolled hypertension, hyperlipidemia, diabetes mellitus, hypercalcemia, and hypothyroidism that presented to the emergency room for weakness and somnolence. On further workup, the patient was found to have a $12-\mathrm{cm}$ neoplasm involving the left upper quadrant. The patient underwent surgical resection of the mass followed by left adrenalectomy. During surgical exploration, multiple tiny lesions were noted while examining the liver (Fig. 1). A right hepatic lobe wedge biopsy was performed and sent to pathology. Pathology revealed stage 4 ACC with hepatic metastatic disease. Lymph node and peritoneal biopsies were negative for malignant cells.

The liver metastatic disease was treated medically. A Port-a-Cath was placed, and the patient was treated with mitotane and 3 cycles of etoposide, Adriamycin, and cisplatin, as well as with metyrapone for blood pressure control. A follow-up CT scan at 3 months then showed new/enlarged liver metastatic disease while the patient was undergoing chemotherapy. After 
Pigg et al.: Y-90 Radioembolization of Metastatic Adrenocortical Carcinoma to the Liver

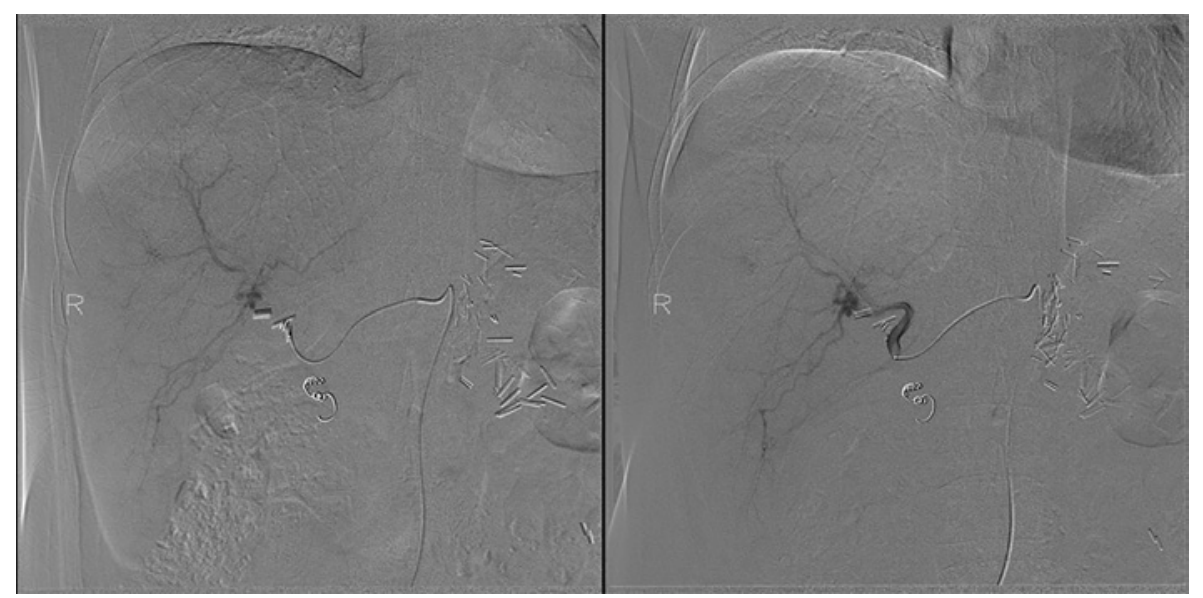

Fig. 2. Fluoroscopic imaging of Y-90 radioembolization of the lesions of the right lobe of the liver after embolization of the gastroduodenal artery.

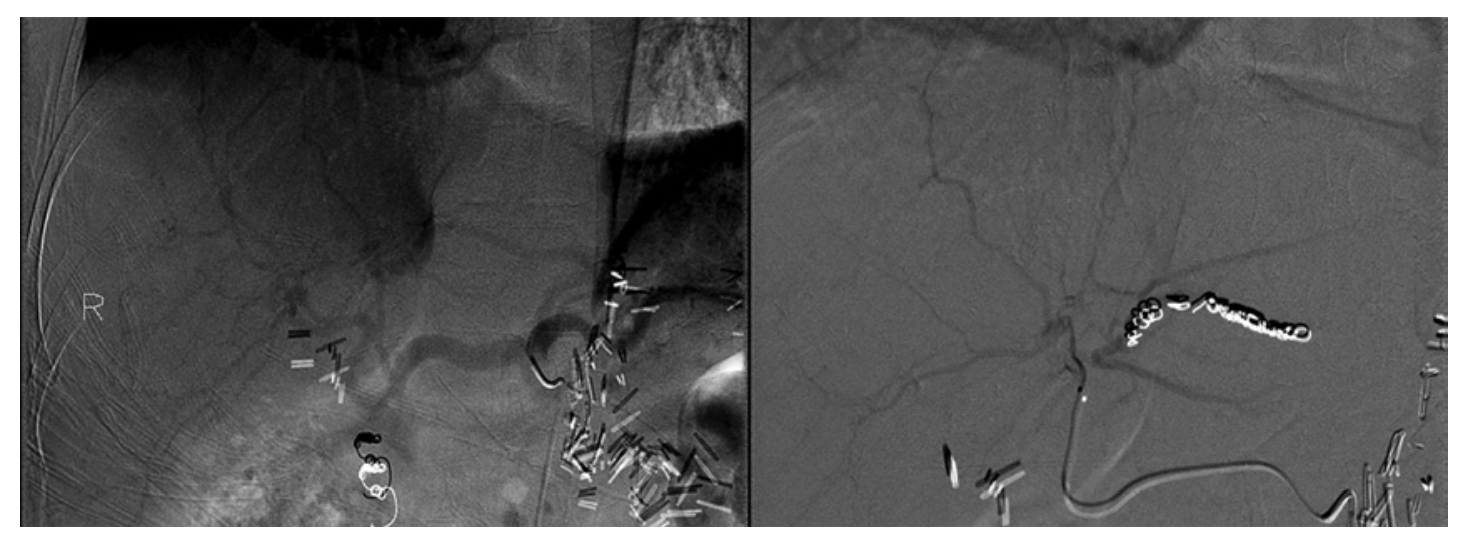

Fig. 3. Fluoroscopic imaging of Y-90 radioembolization of the lesions of the left lobe of the liver following embolization of the left gastrohepatic trunk. Notice the redistribution of flow from the left hepatic artery to segments 2 and 3 .

radiographic evidence of progression of disease and chemotherapy failure, palliative care consideration began. After a multidisciplinary tumor board meeting, we considered possible treatment of the metastatic liver disease with intra-arterial radioembolization.

The patient underwent 2 radioembolization treatments. After embolization of the gastroduodenal artery, the first Y-90 radioembolization targeted the lesions within the right lobe of the liver (Fig. 2). Then, the second Y-90 treatment targeted the left hepatic lobe, following embolization of the left gastrohepatic trunk to prevent collateral flow of treatment (Fig. 3).

The expected outcome of the treatment was tumor debulking for palliative measures. After Y-90 treatment, the patient was followed up at the interventional clinic for 2 years and received follow-up MRI. Initial imaging showed a decreased tumor size and lack of enhancement (Fig. 4, 5). Two years after the treatment, imaging showed numerous stable nonenhancing lesions on the portal venous phase, imperceptible on the delayed phase and also on the noncontrast phases, consistent with treated nonviable lesions. The patient has since been symptom free and has shown no evidence of recurrence. 
Pigg et al.: Y-90 Radioembolization of Metastatic Adrenocortical Carcinoma to the Liver

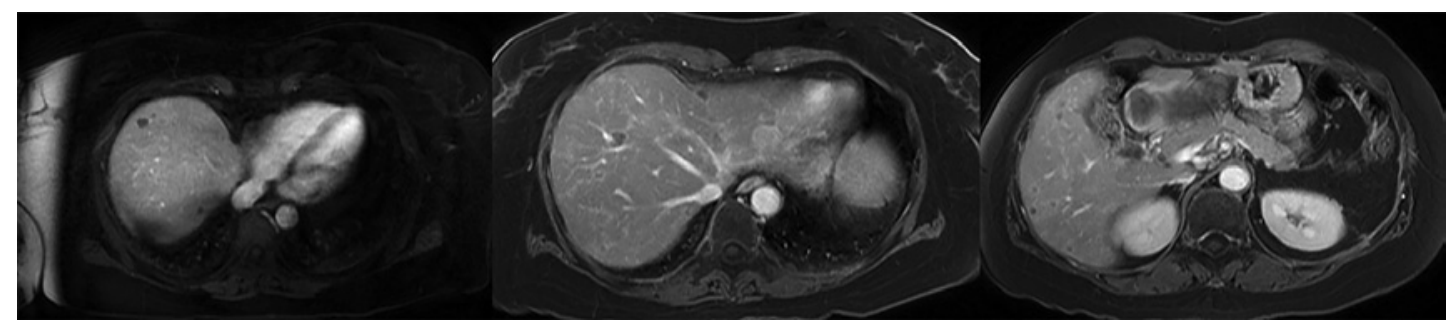

Fig. 4. Axial contrast-enhanced MR images from 2 years after treatment revealing numerous stable nonenhancing structures on the portal venous phase, imperceptible on the delayed phase and also on the noncontrast phases. This is favored to represent a response to treatment in this patient with a history of metastatic liver disease.
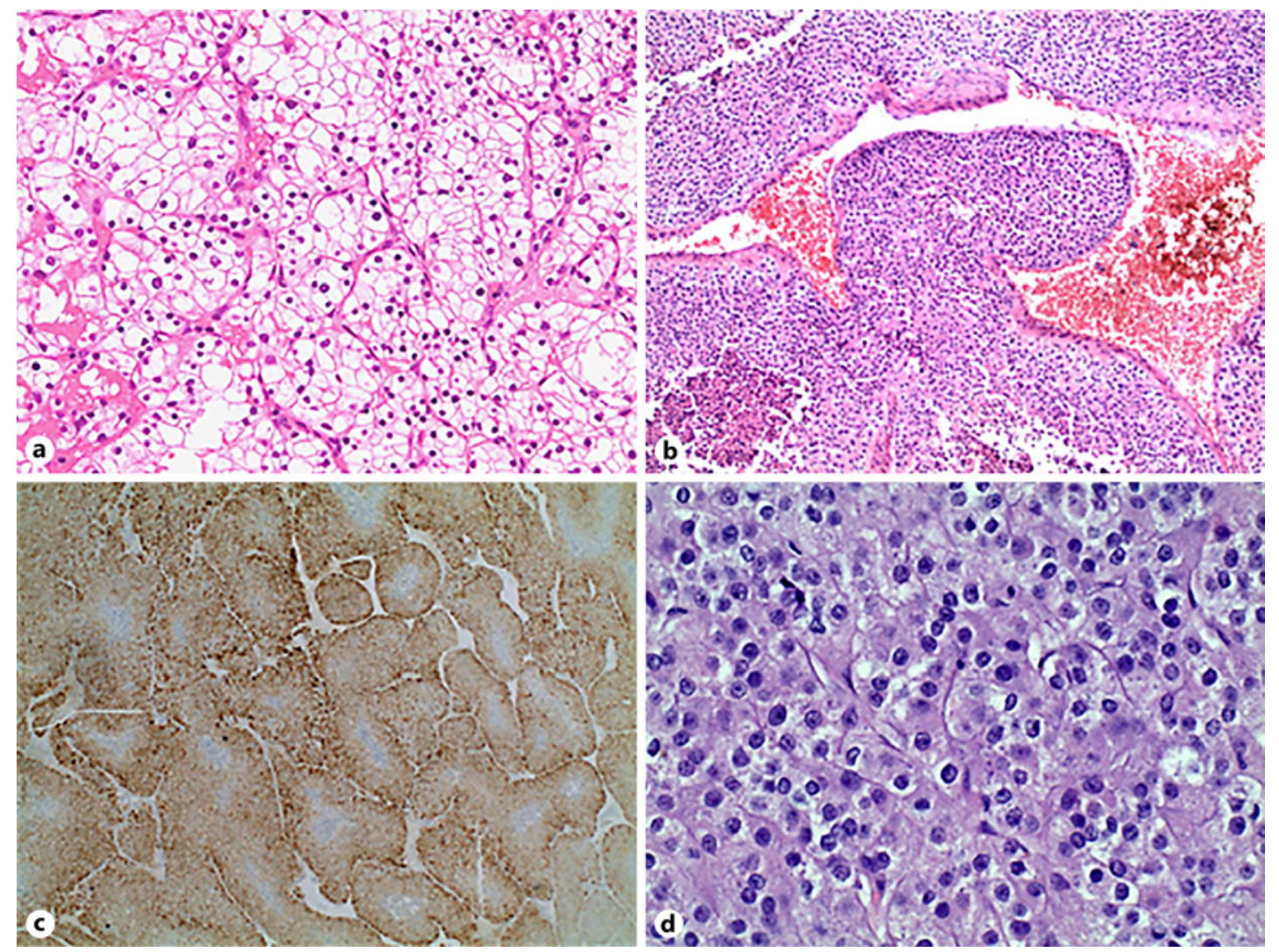

Fig. 5. a Microscopic metastasis of adrenocortical adenocarcinoma (ACC) to the liver. b Vascular intrusion of the tumor with focal tumor necrosis. c Calretinin-positive tumor cells. $\mathbf{d}$ Microscopic metastasis of the ACC. $\times 400$.

\section{Discussion}

ACC is a rare malignancy with a reported incidence of approximately 1 per 1 million [5]. The presence of distant metastatic disease contributes to a significantly worsened clinical outcome. Maximal 5-year survival rates for stage 4 ACC have been reported to be up to 7\% [5]. Unfortunately, due to the lack of early clinical symptoms, only about $30 \%$ of ACC are 
detected prior to extra-adrenal involvement [6]. Furthermore, chemotherapy with mitotane, used as the mainstay treatment, yields response rates of only up to 31\% [7]. Treatment of metastatic liver disease with surgical resection, percutaneous ablation, and endovascular chemoembolization has also been attempted, with variable success rates debated [8]. Clearly, these techniques would prove of limited use in the setting of diffuse liver metastatic disease.

The use of Y-90 for metastatic ACC liver disease as in this case is currently off-label. We came across only one such documented case report with similar positive results in the management of metastatic ACC to the liver refractory to chemotherapy [4]. In this case report, we described a 52-year-old man with stage 4 ACC and extensive liver metastatic disease. After failed chemotherapy with progression of disease, a multidisciplinary tumor board was held, and Y-90 treatment targeting the liver metastatic disease was suggested. The purpose of Y-90 was thought to provide some palliative benefit to the patient. After undergoing bilobar Y-90 treatment, the patient demonstrated a marked clinical and radiographic response. The performance status improved dramatically from ECOG 3 to ECOG 0 [4]. This case report, along with the similar previously reported case, demonstrates a key role for Y-90 therapy in targeting ACC liver metastatic disease.

\section{Conclusion}

Currently, ACC with liver metastatic disease has a very poor prognosis with a poor clinical response to systemic chemotherapy. Other treatment options such as surgical resection, percutaneous ablation, and endovascular chemoembolization have variable success rates. In our case, Y-90 radioembolization elicited a marked response with no evidence of recurrence for 3 years. This case, along with a single similar previously described case, provides strong evidence for the potential of Y-90 as a mainstay of treatment for metastatic ACC liver disease.

\section{Statement of Ethics}

The published research complied with the guidelines for human studies and was conducted ethically in accordance with the World Medical Association Declaration of Helsinki. The subject gave written informed consent to publish her case, including publication of images. IRB approval was not required in this case.

\section{Disclosure Statement}

The authors report no conflict of interest concerning the materials or methods used in this case report. This research was supported (in whole or in part) by HCA and/or an HCAaffiliated entity. The views expressed in this publication represent those of the author(s) and do not necessarily represent the official views of HCA or any of its affiliated entities.

\section{Funding Sources}

There were no external sources of financial support for this manuscript. 
Pigg et al.: Y-90 Radioembolization of Metastatic Adrenocortical Carcinoma to the Liver

\section{Author Contributions}

All authors made significant contributions to the project.

\section{References}

1 Saini A, Wallace A, Alzubaidi S, Knuttinen MG, Naidu S, Sheth R, et al. History and evolution of yttrium-90 radioembolization for hepatocellular carcinoma. J Clin Med. 2019;8(1):E55.

2 Park JK, Phyu W, Zaw T, Walsworth M, Lee HY, Lee EW. Yttrium-90 radioembolization of nonconventional liver tumors. Intervent Oncol. 2016;4(7):E115-E129.

3 Allolio B, Fassnacht M. Clinical review: adrenocortical carcinoma: clinical update. J Clin Endocrinol Metab. 2006;91(6):2027-37.

4 Makary MS, Krishner LS, Wuthrick EJ, Bloomston MP, Dowell JD. Yttrium-90 microsphere selective internal radiation therapy for liver metastases following systemic chemotherapy and surgical resection for metastatic adrenocortical carcinoma. World J Clin Oncol. 2018;9(1):20-5.

5 Sharma E, Dahal S, Sharma P, Bhandari A, Gupta V, Amgai B, et al. The characteristics and trends in adrenocortical carcinoma: a United States population based study. J Clin Med Res. 2018;10(8):636-40.

6 Libé R. Adrenocortical carcinoma (ACC): diagnosis, prognosis, and treatment. Front Cell Dev Biol. 2015;3:45.

7 Fay AP, Elfiky A, Teló GH, McKay RR, Kaymakcalan M, Nguyen PL, et al. Adrenocortical carcinoma: the management of metastatic disease. Crit Rev Oncol Hematol. 2014;92(2):123-32.

8 Zurkiya O, Ganguli S. Beyond hepatocellular carcinoma and colorectal metastasis: the expanding applications of radioembolization. Front Oncol. 2014;4:150. 